\title{
Disruption time scales of star clusters in different galaxies
}

\author{
H. J. G. L. M. Lamers ${ }^{1}$, M. Gieles ${ }^{1}$, and S. F. Portegies Zwart ${ }^{2,3}$ \\ 1 Astronomical Institute, Utrecht University, Princetonplein 5, 3584CC Utrecht, The Netherlands \\ e-mail: [lamers;gieles]@astro.uu.nl \\ 2 Astronomical Institute, University of Amsterdam, Kruislaan 403, 1098SJ Amsterdam, The Netherlands \\ e-mail: spz@science.uva.nl \\ 3 Informatics Institute, University of Amsterdam, Kruislaan 403, 1098SJ Amsterdam, The Netherlands
}

Received 15 June 2004 / Accepted 2 September 2004

\begin{abstract}
The observed average lifetime of the population of star clusters in the Solar Neighbourhood, the Small Magellanic Cloud and in selected regions of M 51 and M 33 is compared with simple theoretical predictions and with the results of $\mathrm{N}$-body simulations. The empirically derived lifetimes (or disruption times) of star clusters depend on their initial mass as $t_{\mathrm{dis}}{ }^{\text {emp }} \propto M_{\mathrm{cl}}{ }^{0.60}$ in all four galaxies. $N$-body simulations have shown that the predicted disruption time of clusters in a tidal field scales as $t_{\mathrm{dis}}{ }^{\text {pred }} \propto t_{\mathrm{rh}}^{0.75} t_{\mathrm{cr}}^{0.25}$, where $t_{\mathrm{rh}}$ is the initial half-mass relaxation time and $t_{\mathrm{cr}}$ is the crossing time for a cluster in equilibrium. We show that this can be approximated accurately by $t_{\mathrm{dis}}$ pred $\propto M_{\mathrm{cl}}^{0.62}$ for clusters in the mass range of about $10^{3}$ to $10^{6} M_{\odot}$, in excellent agreement with the observations. Observations of clusters in different extragalactic environments show that $t_{\mathrm{dis}}$ also depends on the ambient density in the galaxies where the clusters reside. Linear analysis predicts that the disruption time will depend on the ambient density of the cluster environment as $t_{\mathrm{dis}} \propto \rho_{\mathrm{amb}}^{-1 / 2}$. This relation is consistent with $N$-body simulations. The empirically derived disruption times of clusters in the Solar Neighbourhood, in the SMC and in M 33 agree with these predictions. The best fitting expression for the disruption time is $t_{\mathrm{dis}}=C_{\mathrm{env}}\left(M_{\mathrm{cl}} / 10^{4} M_{\odot}\right)^{0.62}\left(\rho_{\mathrm{amb}} / M_{\odot} \mathrm{pc}^{-3}\right)^{-0.5}$ where $M_{\mathrm{cl}}$ is the initial mass of the cluster and $C_{\text {env }} \simeq 300-800$ Myr. The disruption times of star clusters in M 51 within $1-5 \mathrm{kpc}$ from the nucleus, is shorter than predicted by about an order of magnitude. This discrepancy might be due to the strong tidal field variations in M 51, caused by the strong density contrast between the spiral arms and interarm regions, or to the disruptive forces from giant molecular clouds.
\end{abstract}

Key words. Galaxy: open clusters and associations: general - galaxies: individual: M 33 - galaxies: individual: M 51 galaxies: individual: SMC - galaxies: star clusters - Galaxy: globular clusters: general

\section{Introduction}

The age distribution of star clusters in the disk of the Milky Way, can only be explained if galactic clusters disrupt on a time scale on the order of a few times $10^{8}$ years (Oort 1957; Wielen 1971, 1988). Clusters in the LMC and SMC survive longer than those in the Solar neighborhood (e.g. Elson \& Fall 1985, 1988; Hodge 1987).

The disruption time of clusters is expected to depend on both the internal cluster conditions, such as the initial mass, density and velocity dispersion and the stellar initial mass function (IMF), and on the external conditions, such as the orbit in the galaxy and tidal heating by encounters with for example giant molecular clouds. Recently, Boutloukos \& Lamers (2003, hereafter called BL03) have derived an empirical expression for the disruption time of clusters as a function of their initial mass in selected regions in four galaxies. They found large differences in the disruption times between these regions. This allows a crucial test of the theoretical disruption times of clusters predicted with $N$-body simulations.
The purpose of this paper is three-fold:

(a) to explain the empirical dependence of the cluster disruption times on cluster mass;

(b) to explain the strong dependence, found by BL03 of the disruption time on the conditions in the host galaxies;

(c) to confront the predicted disruption theory with the empirically derived disruption relations.

In Sect. 2 we describe the predicted dependence of the disruption times of star clusters on their initial mass and on their environment. In Sect. 3 we review the method used for the determination of the disruption times based on a statistical analysis of large cluster samples and the resulting expressions for the disruption times in four galaxies. In Sect. 4 we discuss the observed dependence of the disruption times on the environment of the clusters in their host galaxies and we compare it with the predictions. We discuss the peculiar case of the clusters in M 51. The conclusions are in Sect. 5. 


\section{The predicted dependence of the disruption time on cluster parameters and environment}

The evolution of an isolated star cluster is characterized by three distinct phases: (1) infant mortality (the dissolution of unbound clusters) within about $10 \mathrm{Myr}$ (Bastian et al. 2005; Whitmore 2004), (2) stellar evolution dominates the cluster mass loss in the first $\sim 10^{8}$ years (see Takahashi \& Portegies Zwart 2000) followed by (3) a relaxation dominated phase in which the cluster mass loss is driven by its internal dynamical evolution and the external influence of the tidal field of the host galaxy. If the cluster survives the first two phases, its lifetime will be dominated by the third phase as it generally lasts much longer, until complete dissolution. During this episode mass loss is about constant with time and appears to depend weakly on the density profile of the cluster (Spitzer 1987; Portegies Zwart et al. 2001b, but see Portegies Zwart et al. 1998, for some complications near the end of the lifetime of the star cluster). During the third phase there are two important effects which drive the cluster dissolution. These are internal two-body relaxation and the interaction between the cluster and the tidal field of the host Galaxy.

Internal two-body relaxation drives the cluster evaporation on a very long time scale of many initial half-mass relaxation times (Baumgardt et al. 2002). The half-mass relaxation time scale can be written as (Spitzer 1958):

$t_{\mathrm{rh}}=0.138 \frac{N^{1 / 2} r_{\mathrm{hm}}^{3 / 2}}{\langle m\rangle^{1 / 2} G^{1 / 2} \ln \Lambda} \propto \frac{N}{\ln \Lambda}\left(\frac{r_{\mathrm{hm}}{ }^{3}}{G M_{\mathrm{cl}}}\right)^{1 / 2}$

where $N$ is the initial number of stars in the clusters, $\langle m\rangle=$ $M_{\mathrm{cl}} / N$ is the mean mass of the stars and $r_{\mathrm{hm}}$ is the half-mass radius. The Coulomb logarithm $\Lambda$ is approximately proportional to $N$ (see Gierz \& Heggie 1996; Spinnato et al. 2003).

The mass loss rate for clusters in a tidal field is much higher and the lifetime shorter than for clusters in isolation. Baumgardt (2001) found that for clusters in a tidal field the disruption time depends not only on the relaxation time but also on the crossing time $t_{\text {cr }}$ because, due to their circulation within the cluster, stars with sufficient energy still need a considerable time to reach the outskirts of the cluster from where they can escape. He showed that the disruption time can be approximated as

$t_{\mathrm{dis}} \simeq t_{\mathrm{rh}}^{x} t_{\mathrm{cr}}^{1-x}$

where the crossing time for a cluster in equilibrium is

$t_{\mathrm{cr}} \propto r_{\mathrm{hm}}^{3 / 2} / \sqrt{G M_{\mathrm{cl}}}$.

and so $t_{\mathrm{cr}} \propto t_{\mathrm{rh}} \ln \Lambda / N$.

Baumgardt (2001) showed on theoretical arguments that $x$ is expected to be about 3/4. A more accurate estimate of the value of $0<x<1$ can be found empirically from $N$-body simulations (see below). Combining Eqs. (1)-(3) an expression for the disruption time can be derived of the form

$t_{\mathrm{dis}}=C\left[\frac{N}{\ln (\gamma N)}\right]^{x}\left[\frac{r_{\mathrm{hm}}{ }^{3}}{G M_{\mathrm{cl}}}\right]^{1 / 2}$

where we replaced $\Lambda$ with $\gamma N$, with $0.01<\gamma<0.4$.

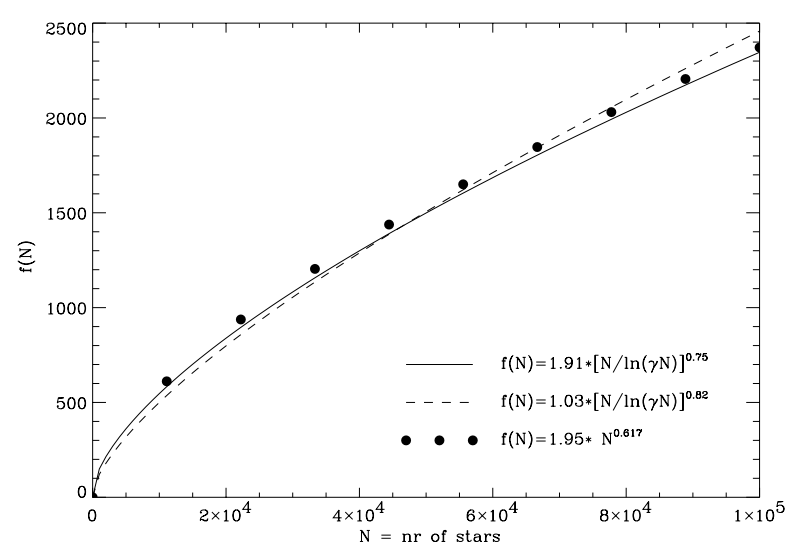

Fig. 1. The function $\beta\{N / \ln (0.02 N)\}^{x}$ of Eq. (6) for the two combinations of $\beta$ and $x$ for clusters with $W_{0}=5$ (solid line) and 7 (dashed) from BM03 and the power law approximation $1.95 N^{0.617}$ (dots).

Baumgardt \& Makino (2003, hereafter BM03) have performed an extensive set of simulations of clusters in the Galactic halo tidal field and fitted the results to find the constant $C$ and the value of $x$ in the approximation of Eq. (4). They used a logarithmic potential of the form $\phi\left(R_{\mathrm{G}}\right)=V_{\mathrm{G}}^{2} \ln \left(R_{\mathrm{G}}\right)$, where $R_{\mathrm{G}}$ is the distance to the galactic center and $V_{\mathrm{G}}$ is the circular velocity. Their clusters started with a tidal radius equal to the tidal radius of the external field,

$r_{\mathrm{t}}=\left(\frac{G M_{\mathrm{cl}}}{2 V_{\mathrm{G}}^{2}}\right)^{1 / 3} R_{\mathrm{G}}^{2 / 3}$.

If we assume that the tidal radius scales linearly with the halfmass radius, which holds for clusters with the same density profile, Eq. (5) can be combined with Eq. (4), which yields

$\frac{t_{\mathrm{dis}}}{\mathrm{Myr}}=\beta\left[\frac{N}{\ln (\gamma N)}\right]^{x} \frac{R_{\mathrm{G}}}{\mathrm{kpc}}\left(\frac{V_{\mathrm{G}}}{220 \mathrm{~km} \mathrm{~s}^{-1}}\right)^{-1}$

where $\beta$ is a constant whose value can be found empirically.

BM03 fitted this relation to the results of their simulations of clusters at different distances from the Galactic center. For clusters that initially followed a King (1966) profile with central concentration $W_{0}=5.0$ they found $\beta=1.91$ and $x=0.75$. For clusters with $W_{0}=7.0$ they found $\beta=1.03$ and $x=0.82$. For $\gamma$ a value of 0.02 was adopted. We found that the factor $\beta\{N / \ln (0.02 N)\}^{x}$ in Eq. (6) for both combinations of $\beta$ and $x$ can be approximated by a power law $1.95 N^{0.62}$ with high accuracy. This is illustrated in Fig. 1.

The density of the clusters depends on the ambient density which depends on the adopted potential field. The ambient density, for the potential field adopted in the study by BM03 can be found by applying Poisson's law which gives

$\rho_{\mathrm{amb}}=\frac{1}{4 \pi G}\left(\frac{V_{\mathrm{G}}}{R_{\mathrm{G}}}\right)^{2}$.

Combining this with Eq. (6), and assuming an initial mean stellar mass of $\langle m\rangle=0.54 M_{\odot}$, as done by BM03 based on the stellar IMF of Kroupa (2001), we can write the disruption time as a function of cluster mass and local ambient density:

$t_{\text {dis }} \simeq 810\left(\frac{M_{\mathrm{cl}}}{10^{4} M_{\odot}}\right)^{0.62} \rho_{\mathrm{amb}}^{-1 / 2} \quad \mathrm{Myr}$ 


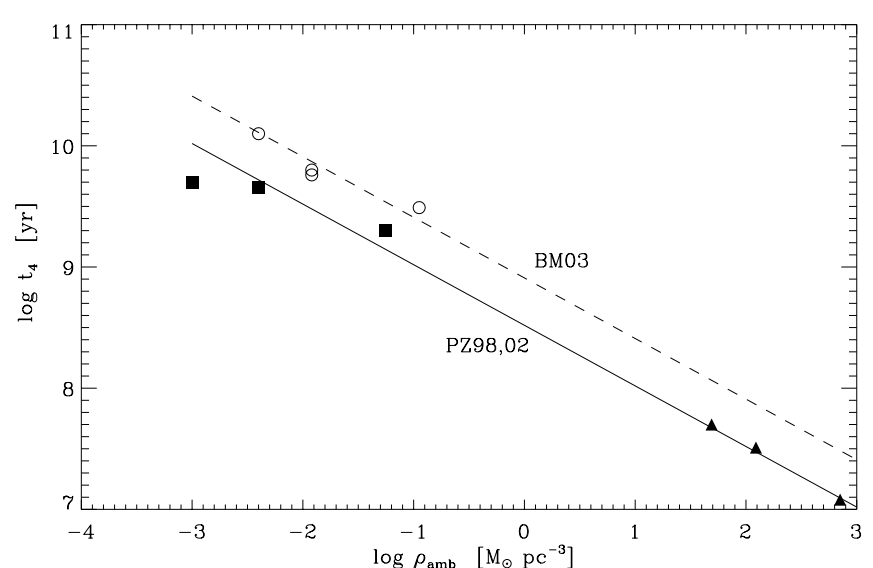

Fig. 2. The predicted dependence of the disruption time, $t_{4}$, of clusters of initial mass of $10^{4} M_{\odot}$ on the mean ambient density, $\rho_{\text {amb }}$. The symbols refer to $N$-body simulations; filled squares are from Portegies Zwart et al. (1998, from left to right models IR16, IC16 and FH16), filled triangles are calculations for star clusters near the Galactic center from Portegies Zwart et al. (2002, from left to right models R150W4, R90W4 and R34W4). The open circles represent models from BM03 (from left to right models V, I, II and IV with 16384 stars). The dashed line is the relation predicted by BM03, Eq. (8), and the full line is the relation predicted by Portegies Zwart et al.

with $M_{\mathrm{cl}}$ in units of $M_{\odot}$ and $\rho_{\mathrm{amb}}$ in $M_{\odot} \mathrm{pc}^{-3}$. The constant indicates the time when 95 per cent of the initial cluster mass is lost. If the initial mean stellar mass is $0.70 M_{\odot}$, predicted by the stellar IMF from Scalo (1986), the constant decreases from 810 to 690. (For clusters in elliptical orbits with ellipticity $\epsilon$ moving within a logarithmic potential field the disruption time is shorter by factor $1-\epsilon$, if $\rho_{\mathrm{amb}}$ is the density at the apogalactic radius.) The positive exponent 0.62 indicates that the disruption time increases with mass, as expected.

The results of $N$-body simulations and their predicted dependence on the ambient density are plotted in Fig. 2. These are based on $N$-body simulations of clusters of $10^{4} M_{\odot}$ from different authors. These simulations are from Portegies Zwart et al. (1998, 2002), and BM03. All simulations were performed with NBODY4 (Aarseth 1999; BM03) and the Starlab (Portegies Zwart et al. 2001a) ${ }^{1}$ software environment with both codes running on the GRAPE family of special purpose computers (Makino 2003). The models by Portegies Zwart et al. (2002) were calculated for star clusters near the Galactic center, i.e. they are representative for a high density ambient medium.

The dashed line shown in this figure represents the mean relation derived by BM03 and described by Eq. (8). This line passes through the data of their individual models.

The full line is the mean relation predicted by Portegies Zwart et al. (2001b). It is derived from the argument that the lifetime of a cluster will depend on the two-body relaxation time near the tidal radius as $t_{\mathrm{dis}} \simeq 0.3 t_{\mathrm{rt}}$. The tidal radius can be obtained from Eq. (5), but the required average cluster density $\rho_{\mathrm{cl}}$ is not readily available from the observations. Since the cluster is in equilibrium with its environment one can estimate $\rho_{\mathrm{cl}} / \rho_{\mathrm{amb}} \simeq 3$ for a standard

\footnotetext{
1 see also http://www. manybody.org
}

Roche-solution by assuming that the clusters follow a King (1966) model and that the tidal radius of the King model equals the Jacobi radius of the star cluster in the tidal field of the Galaxy (see e.g. Binney \& Tremaine 1994). This predicts that the constant in Eq. (8) is about 330 Myr. This is 0.4 times as small as the value derived from the models by BM03. The line passes through the data of the individual models of Portegies Zwart et al. (1998, 2002).

The simulations by Portegies Zwart et al. (1998, 2002) result in shorter disruption times than the simulations by BM03, by about a factor of 2.5. This difference can be explained by variations in the initial conditions and between the two codes. The initial mass function used in Portegies Zwart et al. (2002) is taken from Scalo (1986) and has a mean stellar mass of $\langle m\rangle \simeq 0.7 M_{\odot}$. BM03 adopted the initial mass function of Kroupa (2001), which has a mean stellar mass of $0.54 M_{\odot}$. Portegies Zwart et al. (1998) and BM03 use King (1966) models for the initial density distribution, whereas Portegies Zwart et al. (2002) adopt self consistent Heggie \& Ramamani (1995) models. But also the stellar evolution in the calculations are slightly different: the stellar evolution in BM03 is based on Pols et al. (1998) whereas the calculations of Portegies Zwart et al. use the stellar evolution predicted by Eggleton et al. (1989). Moreover, the end of the cluster lifetime is defined differently in the various calculations: BM03 and Portegies Zwart et al. (2002) uses the time when 5 percent of the initial mass remains, whereas Portegies Zwart et al. (1998) use the time when 10 percent of the initial mass remains. The most important difference, however, is in the treatments of the tidal field and the way stars escape from the cluster. Portegies Zwart et al. (1998) did not include a full tidal potential, but a simple cut-off radius. This easily leads to a shorter disruption time for the cluster. BM03 and Portegies Zwart et al. (2002), on the other hand adopt a self-consistent tidal field up to the quadrupole moment ${ }^{2}$.

A few notes about these estimate:

(a) The constant of expression (8) was derived for clusters moving in circular orbits in the Galactic potential field. Clusters in elliptical or inclined orbits will experience disk or bulge shocking that reduces their lifetime (Spitzer 1987; Zhang \& Fall 1999; BM03). However the proportionality with mass and density remains the same.

(b) Encounters with giant molecular clouds (GMCs) or spiral density waves are not included in these calculations. In situations where these effects are likely to occur, e.g. for clusters in orbits in a galactic plane, the constant will be smaller than estimated above.

Based on these considerations we can expect the following expression for the disruption times of star clusters due to tidal interactions in different extragalactic environments

$t_{\mathrm{dis}}=C_{\mathrm{env}}\left(\frac{M_{\mathrm{cl}}}{10^{4} M_{\odot}}\right)^{0.62}\left(\frac{\rho_{\mathrm{amb}}}{M_{\odot} \mathrm{pc}^{-3}}\right)^{-1 / 2}$

${ }^{2}$ In an extensive collaborative experiment Heggie et al. (1998) perform a detailed comparison between the N-body codes used by Portegies Zwart and Baumgardt (and others). The difference in cluster lifetime is between 20 and $40 \%$. (see http: //www . maths.ed.ac.uk/ douglas/experiment.html) 
where $C_{\text {env }} \simeq 300-800$ Myr. If encounters with giant molecular clouds or disk shocking (for clusters in orbits tilted with respect to the galactic plane) becomes important, the disruption times might be shorter than given by Eq. (9).

\section{The empirically determined disruption times}

The disruption times of systems of star clusters in different extragalactic environments have been determined statistically by BL03 from samples of star clusters in four galaxies. We will summarize their method and results.

Suppose that the disruption times of clusters in some region of a galaxy can be written as

$t_{\mathrm{dis}}=t_{4} \times\left(M_{\mathrm{cl}} / 10^{4}\right)^{\gamma}$

where $M_{\mathrm{cl}}$ is the initial cluster mass (in $M_{\odot}$ ) and $t_{4}$ is the disruption time (in yrs) of a cluster with an initial mass of $M_{\mathrm{cl}}=10^{4} M_{\odot}$. Assume also that the cluster formation rate is constant over the period of time for which $t_{4}$ and $\gamma$ are determined, and that all clusters had the same initial stellar IMF. Then, the values of $\gamma$ and $t_{4}$ can be derived from the mass and age histograms of cluster samples with a well determined brightness limit. Boutloukos \& Lamers (BL03) have applied this method for an empirical determination of $\gamma$ and $t_{4}$ in specific regions of M 51, M 33 (observed with $H S T$ - WFPC2) and in the SMC and the Solar neighbourhood. The method has been improved by Lamers (2004) and by Gieles et al. (2004c), who showed that the distribution of the observed clusters in a mass versus-age-diagram provides a more powerful method for deriving $t_{4}$ and $\gamma$ than the mass and age histograms separately.

The results are given in Table 1. Column 2 gives the range of galactocentric distances of the cluster sample. Columns 3-5 give the number of clusters and their age and mass range. Columns 6 and 7 give the resulting values of the disruption time $t_{4}$ and $\gamma$. Column 8 gives the ambient density of the host galaxy at the location of the cluster samples used for the determination of the disruption time (see Sect. 4).

In the determination of $\gamma$ we adopted a cluster IMF with $\alpha=2.0$ (Zhang \& Fall 1999; Bik et al. 2002; de Grijs et al. 2003). The values of $t_{4}$ and $\gamma$ of the SMC is from BL03. The values for M 51 have been redetermined by Gieles et al. (2004c) on the basis of the extended cluster sample of Bastian et al. (2005) and the improved method mentioned above. The values for M 33 have been redetermined by Lamers et al. (2004b), on the basis of the extended cluster sample by Chandar et al. (2001, 2002). The new value of $t_{4}$ for this galaxy is considerably higher than originally derived by BL03, due to the largely improved cluster sample and a redetermination of the cluster ages. The values for the Solar neighbourhood have been redetermined by Lamers et al. (2004b), based on the cluster sample by Loktin et al. (1994).

Notice that the values of $\gamma$ of the four galaxies are the same, within the uncertainty, but the values of $t_{4}$ differ strongly. The mean value of the empirically determined value of $\gamma$ and its uncertainty is

$\left\langle\gamma_{\mathrm{emp}}\right\rangle=0.60 \pm 0.02$ where 0.02 is the uncertainty in the mean value, which is much smaller than the uncertainty in the individual measurements. This exponent agrees very well with $\gamma_{\text {pred }}=0.62$ predicted by BM03, Eq. (8), on the basis of their numerical simulations. The agreement between the observed and the predicted value of $\gamma$ was first pointed out by Gieles et al. (2004d), but based on slightly different theoretical arguments.

\section{The dependence of the disruption time on the conditions in the host galaxy}

Table 1 shows that the constant $t_{4}$, which is the disruption time of a clusters of $M_{\mathrm{cl}}=10^{4} M_{\odot}$, differs greatly between the regions in the galaxies that were studied. This indicates that the disruption time depends strongly on the local conditions in the host galaxy. In Sect. 2 we have shown that $t_{4}$ is expected to scale with the inverse square-root of the mean density in the host galaxy, if evolutionary mass loss, relaxation driven evaporation and tidal stripping are the dominant mass loss mechanism for clusters. In this section we compare this predicted dependence with the empirically derived values of the disruption time $t_{4}$.

\subsection{The ambient density in the host galaxies}

We estimate the ambient density $\rho_{\mathrm{amb}}$ at the galactocentric distances of the observed environments for which BL03, Gieles et al. (2004c) and Lamers et al. (2004b) determined the value of $t_{4}$.

\section{51:}

The clusters are at galactocentric distances of 1 to $5 \mathrm{kpc}$. The column density of M 51 decreases exponentially as $\sigma=$ $540 \exp (-r / 4.65) M_{\odot} \mathrm{pc}^{-2}$, with $r$ in kpc (Salo \& Laurakainen 2000). The total disk mass within a ring of 1 to $5 \mathrm{kpc}$ is $2.0 \times 10^{10} M_{\odot}$ and the mean surface density in that annulus is $2.6 \times 10^{2} M_{\odot} \mathrm{pc}^{-2}$. Van der Kruit (2002) has shown that spiral galaxies have a vertical scale-height of $1 / 7$ times the radial scale-length. This implies a value of $h_{z}=0.66 \mathrm{kpc}$ and a mean mid-plane density between 1 and $5 \mathrm{kpc}$ of $0.20 M_{\odot} \mathrm{pc}^{-3}$. The structural parameters of M 51 derived by Athanassoula et al. (1987) result in a very similar estimate of the mid-plane density.

\section{33:}

The sample of clusters is located at galactocentric distances between 1 and $5 \mathrm{kpc}$. More than 90 percent of the clusters are between 1 and $4 \mathrm{kpc}$. The column density of M 33 decreases exponentially as $\sigma=525 \exp (-r / 1.45) M_{\odot} \mathrm{pc}^{-2}$, with $r$ in kpc (Athanassoula et al. 1987). The total disk mass within a ring of 1 to $4 \mathrm{kpc}$ is $4.2 \times 10^{9} M_{\odot}$ and the mean surface density is $90 M_{\odot} \mathrm{pc}^{-2}$. Adopting an effective thickness of the disk of $1 / 7$ of the radial scale-length, i.e. $0.21 \mathrm{kpc}$, we find a mean midplane density of $0.22 M_{\odot} \mathrm{pc}^{-3}$.

\section{Solar Neighborhood:}

The mean gas and stellar density in the Solar neighborhood, derived from Hipparcos data is $0.10 \pm 0.01 M_{\odot} \mathrm{pc}^{-3}$ (Holmberg \& Flynn 2000). 
Table 1. The parameters of the disruption time: $t_{\mathrm{dis}}=t_{4} \times\left(M_{\mathrm{cl}} / 10^{4}\right)^{\gamma}$.

\begin{tabular}{|c|c|c|c|c|c|c|c|c|}
\hline Galaxy & $\begin{array}{l}r_{\mathrm{Gal}} \\
\mathrm{kpc}\end{array}$ & $\begin{array}{c}\text { No. } \\
\text { clusters }\end{array}$ & $\begin{array}{c}\text { Age range } \\
\log (\mathrm{yr})\end{array}$ & $\begin{array}{c}\text { Mass range } \\
\log M_{\odot}\end{array}$ & $\begin{array}{c}\log t_{4} \\
\log (\mathrm{yr})^{a}\end{array}$ & $\gamma$ & $\begin{array}{l}\log \rho_{\mathrm{amb}} \\
M_{\odot} \mathrm{pc}^{-3}\end{array}$ & Remark \\
\hline M 51 & $1.0-5.0$ & 1152 & $6.3-9.0$ & $2.6-5.6$ & $7.85 \pm 0.22(7.6)$ & $0.57 \pm 0.10$ & $-0.70 \pm 0.30$ & 2 \\
\hline M 33 & $0.8-5.0$ & 147 & $6.5-10.0$ & $3.6-5.6$ & $8.80 \pm 0.20(8.1)$ & $0.60 \pm 0.15$ & $-0.66 \pm 0.30$ & 3 \\
\hline Galaxy $(d \leq 1 \mathrm{kpc})$ & $7.5-9.5$ & 184 & $7.2-9.5$ & - & $8.75 \pm 0.20(9.0)$ & $0.60 \pm 0.12$ & $-1.00 \pm 0.04$ & 1,4 \\
\hline \multirow[t]{2}{*}{ SMC } & $0-4$ & 314 & $7.6-10.0$ & - & $9.90 \pm 0.20(9.9)$ & $0.61 \pm 0.08$ & $-2.10 \pm 0.30$ & 1 \\
\hline & & & & & Mean & $0.60 \pm 0.02$ & & 5 \\
\hline
\end{tabular}

(a) The value in parenthesis is the original value from BL03.

(1) $t_{4}$ and $\gamma$ from age distribution only.

(2) Improved determination of $t_{4}$ and $\gamma$ by Gieles et al. (2004c), based on new cluster sample of Bastian et al. (2005).

(3) Improved determination of $t_{4}$ and $\gamma$ by Lamers et al. (2004b) based on extended cluster samples by Chandar et al. (2001, 2002).

(4) Improved determination by Lamers et al. (2004a) based on cluster sample of Loktin et al. (1994).

(5) The quoted error is the uncertainty in the mean value.

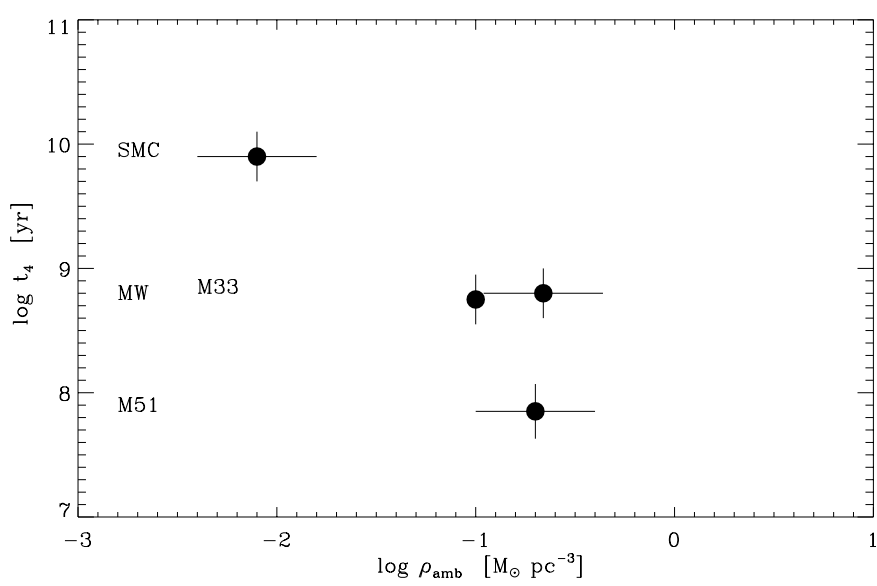

Fig. 3. The relation between the measured values of the disruption time $t_{4}$ of clusters with an initial mass of $10^{4} M_{\odot}$ and the mean ambient density $\rho_{\text {amb }}$ in $M_{\odot} \mathrm{pc}^{-3}$, of the galaxies at the galactocentric distances where the studied clusters were located.

SMC:

The sample of clusters is distributed throughout the SMC. With a total mass of about $2 \times 10^{9} M_{\odot}$ (Mathewson \& Ford 1984) and a radius of the central core of $4 \mathrm{kpc}$ (Caldwell \& Laney 1991) we find a mean density of $8 \times 10^{-3} M_{\odot} \mathrm{pc}^{-3}$.

The ambient densities are listed in Table 1, where we have adopted an uncertainty of a factor 2 in the densities, except for the Solar neighbourhood. The relation between $t_{4}$ and the ambient densities is shown in Fig. 3. The figure shows a clear trend of decreasing disruption time with increasing ambient density.

\subsection{Comparison between observed and predicted disruption time scales}

We compare the observed values of $t_{4}$ for the different galaxies with the predictions based on $\mathrm{N}$-body simulations, discussed in Sect. 2. These simulations were done for clusters in Galactic environments. However, by using the expected scaling laws (Sect. 2), we can apply the results of these simulations to the conditions in the galaxies where the cluster disruption times were measured.

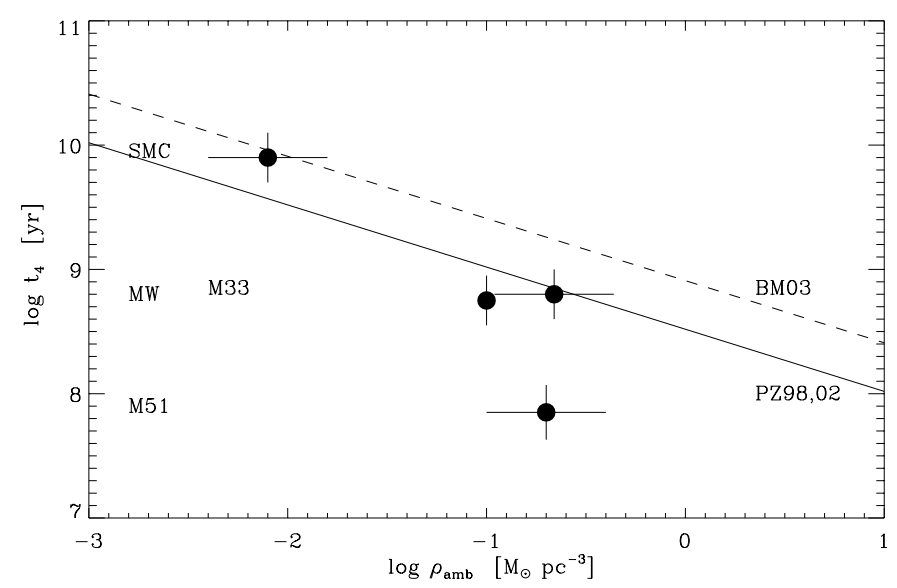

Fig. 4. Comparison between the observed (large dots) and predicted (lines) disruption time $t_{4}$ of clusters with an initial mass of $10^{4} M_{\odot}$ as a function of the mean density $\rho_{\mathrm{amb}}$ in $M_{\odot} \mathrm{pc}^{-3}$ of the host galaxy. The solid curve present the predictions by Portegies Zwart et al. (1998, 2002) and the dashed line by BM03.

In Fig. 4 we compare the observed relation between $t_{4}$ and $\rho_{\text {amb }}$ with the predicted mean relations derived from $N$-body simulations of clusters of $10^{4} M_{\odot}$ from Portegies Zwart (1998, 2002) and BM03, shown in Fig. 2.

The empirically derived values of $t_{4}$ roughly follow the relations predicted by the $N$-body simulations within the uncertainty of the data, except for the clusters in M 51, whose disruption time is almost an order of magnitude shorter than expected.

There are several possible explanations for the short cluster lifetimes in M 51, some of which originate from variations in the birth conditions of the clusters or from star formation histories of the host galaxy. For example, the constant used in Eq. (9) depends via $\langle m\rangle$, quite sensitively on the adopted initial mass function. If we adopt a Salpeter (1955) initial mass function with a lower mass limit of $1 M_{\odot}$, instead of $0.1 M_{\odot}$, the mean stellar mass increases by about an order of magnitude. Such dramatic change to the initial mass function results via Eqs. (4) in a reduction of the cluster lifetime by about a factor 3. Such a lower mass cut-off for the initial mass function is controversial, but is suggested in young star clusters like MGG-11 
(McCrady et al. 2003) and MGG-F, both in M 82 (Smith \& Gallagher 2001) and in several of the young star clusters in the Antennae galaxies (Mengel et al. 2002).

Since M 51 experienced a recent star formation event, about 60 Myr ago (Bastian et al. 2005), possibly part of the discrepancy can be explained by variations in the IMF. (This starburst event was taken into account in the determination of the disruption time for M 51 by Gieles et al. 2004b). Young star formation environments also tend to have higher metalicity, which drives stronger winds and therefore more stellar mass loss. Another possible explanation for the short disruption time of clusters in $\mathrm{M} 51$ is related to the strength of the tidal field variations. M 51 has the highest density contrast between the arm and interarm regions of any spiral galaxy (Rix \& Rieke 1993), so the tidal field variations experienced by star clusters will be higher than normal, which might explain the short disruption time of the star clusters in M 51. This last effect is studied by Gieles et al. (2004a).

\section{Conclusions}

We analyzed the disruption time of star clusters in the Solar Neighbourhood, the SMC and in selected regions of M 33 and M 51. We find the following result.

(a) Within each region, there is a clear relation between the empirically derived cluster disruption time and the cluster mass of the type $t_{\text {dis }} \propto M^{\gamma}$. The mean empirical value of $\gamma_{\mathrm{emp}}=0.60 \pm 0.02$ agrees very well with the predicted value of $\gamma_{\text {pred }}=0.62$, derived from direct $N$-body simulations of galactic star clusters.

(b) Comparing the disruption times in different galaxies, we find that the disruption time depends on the ambient density in the environment where the clusters reside. $N$-body simulations of clusters at different galactocentric distances predict that the disruption time decreases with the ambient density as $t_{\mathrm{dis}} \propto \rho_{\mathrm{amb}}{ }^{-0.5}$. The empirical values of three galaxies, SMC, M 33 and the Solar neighbourhood agree roughly with this prediction. However, the disruption time of star clusters in the inner $4 \mathrm{kpc}$ of M 51 is shorter than predicted by about an order of magnitude. The difference is most likely due to large tidal field variations, due to the high density contrast in the spiral arms and interarm regions of M 51, or due the starburst triggered by the last encounter with its companion NGC 5195.

The disruption time of star clusters of initial mass $M_{\mathrm{cl}}$ in circular orbits in an environment with a mean ambient density $\rho_{\mathrm{amb}}$ is given by Eq. (9). The disruption time may be shorter in starburst regions.

Acknowledgements. We thank Nate Bastian for discussions and comments on the manuscript, Albert Bosma and Piet van der Kruit for advice on the densities of spiral galaxies and Holger Baumgardt for providing the results of his numerical simulations. We are grateful to the referee, Douglas Heggie, for constructive suggestions that improved the paper. This work was supported by a grant from the Netherlands
Research School for Astronomy (NOVA) to HJGLML and by a grant from the Royal Netherlands Academy of Sciences (KNAW) to SPZ.

\section{References}

Aarseth, S. J. 1999, PASP, 111, 1333

Athanassoula, E., Bosma, A., \& Papaioannou, S. 1987, A\&A, 179, 23

Bastian, N., Gieles, M., Lamers, H. J. G. L. M., de Grijs, R., \& Scheepmaker, R. 2005, A\&A, in press

Baumgardt, H. 2001, MNRAS, 325, 1323

Baumgardt, H., \& Makino, J. 2003, MNRAS, 340, 227 (BM03)

Baumgardt, H., Hut, P., \& Heggie, D. C. 2002, MNRAS, 336, 1069

Bik, A., Lamers, H. J. G. L. M., Bastian, N., Panagia, N., \& Romaniello, M. 2002, A\&A, 397, 473

Binney, J., \& Tremaine, S. 1994, Galactic Dynamics (Princeton: Princeton University Press)

Boutloukos, S. G., \& Lamers, H. J. G. L. M. 2003, MNRAS, 338, 717 (BL03)

Chandar, R., Bianchi, L., \& Ford, H. C. 2001, A\&A, 366, 498

Chandar, R., Bianchi, L., Ford, H. C., \& Sarajedini, A. 2002, ApJ, 564, 712

Caldwell, J. A. R., \& Laney, C. D. 1991, in The Magellanic Clouds, ed. R. Haynes, \& D. Milne (Dordrecht: Kluwer), 249

de Grijs, R., Anders, P., Bastian, N., et al. 2003, MNRAS, 343, 1285

Eggleton, P. P., Fitchet, M., \& Tout, C. A. 1989, ApJ, 347, 998

Elson, R. A. W., \& Fall, S. M. 1985, ApJ, 299, 211

Elson, R. A. W., \& Fall, S. M. 1988, AJ, 96, 1383

Gieles, M., Athanassoula, E., \& Portegies Zwart, S. F. 2004a, in preparation

Gieles, M., Bastian, N., \& Lamers, H. J. G. L. M. 2004b, in The formation and evolution of massive young star clusters, ed. H. J. G. L. M. Lamers, A. Nota, \& L. J. Smith, ASP Conf. Ser., 322, 479

Gieles, M., Bastian, N., \& Lamers, H. J. G. L. M. 2004c, in preparation

Gieles, M., Baumgardt, H., Bastian, N., \& Lamers, H. J. G. L. M. 2004d, in The formation and evolution of massive young star clusters, ed. H. J. G. L. M. Lamers, A. Nota, \& L. J. Smith, ASP Conf. Ser., 322, 481

Giersz, M., \& Heggie, D. C. 1996, MNRAS, 279, 1037

Heggie, D. C., \& Ramamani, N. 1995, MNRAS, 272, 317

Heggie, D. C., Giersz, M., Spurzem, R., \& Takahashi, K. 1998, Highlights in Astronomy, 11, 591

Hodge, P. 1987, PASP, 99, 742

Holmberg, J., \& Flynn, C. 2000, MNRAS, 313, 209

King, I. R. 1966, AJ, 71, 64

Kroupa, P. 2001, MNRAS, 322, 231

Lamers, H. J. G. L. M. 2004, in preparation

Lamers, H. J. G. L. M., Bastian, N., Gieles, M., \& Baumgardt, H. 2004a, A\&A, submitted

Lamers, H. J. G. L. M., Gieles, M., \& Seth, A. 2004b, in preparation

Loktin, A. V., Matkin, N. V., \& Gerasimenko, T. P. 1994, Astron. Astrophys. Trans., 4, 153

Makino, J. 2003, PASJ, 55, 1163

Mathewson, D. S., \& Ford, V. L. 1984, in Structure and Evolution of the Magellanic Clouds, ed. S. van den Bergh, \& K. S. de Boer, (Dordrecht: Reidel), 125

McCrady, N., Gilbert, A. M., \& Graham, J. R. 2003, ApJ, 596, 240

Mengel, S., Lehnert, M. D., Thatte, N., \& Genzel, R. 2002, A\&A, 383, 137

Oort, J. H. 1957, in Stellar populations (Rome: Pontifical Academy of Science), discussion in Session on star clusters

Pols, O. R., Schroder, K. P., Hurley, J. R., Tout, C. A., \& Eggleton, P. P. 1998, MNRAS, 298, 525 
Portegies Zwart, S. F., Hut, P., \& Makino, J. 1998, A\&A, 337, 363

Portegies Zwart, S. F., McMillan, S. L. W., Hut, P., \& Makino, J. 2001a, MNRAS, 321, 199

Portegies Zwart, S. F., Makino, J., McMillan, S. L. W., \& Hut, P. 2001b, ApJ, 546, L101

Portegies Zwart, S. F., Makino, J., McMillan, S. L. W., \& Hut, P. 2002, ApJ, 565, 265

Rix, H., \& Rieke, M. J. 1993, ApJ, 418, 123

Salpeter, E. E. 1955, ApJ, 121, 161

Salo, H., \& Laurikainen, E. 2000, MNRAS, 319, 377

Scalo, J. M. 1986, Fund. Cosm. Phys., 11, 1

Smith, L. J., \& Gallagher, J. S. 2001, MNRAS, 326, 1027

Spinnato, P. F., Fellhauer, M., \& Portegies Zwart, S. F. 2003, MNRAS, 344,22
Spitzer, L., Jr. 1958, ApJ, 127, 544

Spitzer, L. Jr. 1987, in Dynamical evolution of globular clusters (Princeton: Princeton University Press), 191

Takahashi, K., \& Portegies Zwart, S. F. 2000, ApJ, 535, 759

van der Kruit, P. C. 2002, in The dynamics, structure \& history of galaxies, ed. G. S. Da Costa, \& H. Jerjen, ASP Conf. Proc., 273, 7

Whitmore, B. 2004, in The formation and evolution of massive young star clusters, ed. H. J. G. L. M. Lamers, A. Nota, \& L. J. Smith, ASP Conf. Ser., 322, 419

Wielen, R. 1971, A\&A, 13, 309

Wielen, R. 1988, The Harow Shapley Symposium on Globular Cluster Systems in Galaxies, ed. J. Grindlay, \& A. G. D. Philip (Dordrecht: Reidel), 393

Zhang, Q., \& Fall, S. M. 1999, ApJ, 527, L81 